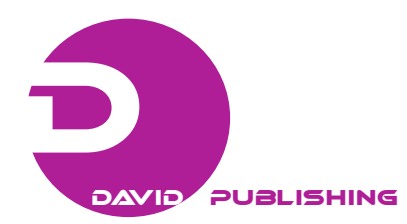

\title{
College Coaching Legends
}

\author{
Xue Yanga, Jiwei Jina
}

\begin{abstract}
Everyone has his heroes, like the college coaches, many of them play a important role in our college life, and they live a legendary life here at the same time. In America, college student pay attention to NCAA (National Collegiate Athletic Association), there are many famous coaches in it, such as Nich Saban, Jerry Tarkanian, and more. The authors are very interested in the competitive life of those who are the top five coaches in the world. So the authors concentrate on the winning rate, the contribution rate, and the cycle of the honors to evaluate each coach, by standardizing the metrics, they get the final scores of each metric for basketball; according to the scores, they get the top five coaches in a century. And in this paper, the authors also take the gender and timeline into account.
\end{abstract}

\section{Keywords}

Standardization of the dispersion, coefficient of variation method, weighted arithmetic mean of comprehensive evaluation

In order to truly achieve evaluating goals, we should uphold the following principles during the modeling process (Ni and Bai 2005; Zhang 2007).

(1) The model should reflect the objectivity and fairness;

(2) Index selection should reflect the comprehensiveness, representativeness, and factuality;

(3) The comprehensive evaluation system of the coach should be feasible and maneuverable, that is, the model should be simple to operate, in time to analyze and easy to manage.

According to the evaluation for sports, the authors make the procedure of the evaluation system. They use questionnaires ${ }^{1}$ on the internet to get the final scores of each metric evaluated on the net. The following is the results.

In Table 1, A represents winning rate, B represents stability, C represents professionalism, D represents Coach of the Year, and E represents Coach of the Year on the net. We can get from Table 1 that people pay more attention to the winning rate, stability, and professionalism, and less attention to the honor of the
Coach of the Year and the Coach of the Year on the net.

\section{DATA STANDARDIZATION}

We suppose that there are $i$ evaluation objects, and $j$ evaluation metrics, the original value of every metric is $x_{i j}$, for positive metrics, general standardization includes linear normalization algorithm and nonlinear normalization algorithm (Yu, Pan, and Wu 2015). The dispersion belongs to the nonlinear normalization algorithm, the computing formula is:

$$
y_{i j}=x_{i j} / \sqrt{\sum_{i=1}^{n} x_{i j}^{2}}
$$

aNorth China Electric Power University, China

\section{Correspondent Author:}

Xue Yang, College of Electrical Engineering and Automation, North China Electric Power University (Baoding), Huadian Road, Beishi district, Baoding, Hebei, 071003, China 
Table 1. The Scores of Five Metrics by Objective Evaluation

\begin{tabular}{llllll}
\hline Metric & A & B & C & D & E \\
\hline Score & 7.5877 & 7.9324 & 7.9726 & 6.0064 & 5.0743 \\
\hline
\end{tabular}

In the equation, the $\mathrm{y}_{i j}$ is the value that is standardized, $\bar{x}_{i j}$ represents the average value of every metric.

\section{DETERMINE THE WEIGHT BY THE COEFFICIENT OF VARIATION METHOD}

Coefficient of variation method directly uses the information included by all the metrics, and get the weight of the metrics through computation, which is a key of objective weight defining. The basic work is: In the evaluation system, the metrics of large difference in values can better reflect the gap of the evaluation. Because the dimensions of every metric in the evaluation system are different, we cannot compare them directly. In order to eliminate the influence of different dimension, we have to use variation coefficient to measure the value of every metric. The variation coefficient formula is as follows:

$$
V_{i}=\sigma_{i} / \bar{x}_{i}(i=1,2, \cdots, n)
$$

In the equation, $V_{i}$ is the variation coefficient for the metric of $i$, or the coefficient of standard deviation. $\sigma_{i}$ is the standard deviation for the metric of $i . \bar{x}_{i}$ is the average number for the metric of $i$.

The formula for the weight of every metric is:

$$
W_{i}=V_{i} / \sum_{i=1}^{n} V_{i}
$$

\section{WEIGHTED ARITHMETIC MEAN OF COMPREHENSIVE EVALUATION}

When using comprehensive evaluation, if the weight of every metric is equal, we can choose the simple average method directly. The basic formula for computing of the weighted arithmetic mean of comprehensive evaluation is as follows:

$$
f=\sum_{i=1}^{n} x_{i} W_{i} / \sum_{i=1}^{n} W_{i}
$$

In the formula, $n$ is the number of the metrics; $x_{i}$ is the relative value for the metric of $i$ under the same measurement, and let $x_{i}$ be a sequence, where $i=1,2,3, \cdots, n ; W_{i}$ is the weight of every metric, that is, $W_{1}+W_{2}+\cdots+W_{n}=100 \%$, similarly, let $W_{i}$ be a sequence.

The feature of the approach is independence of every metric, linear compensation among every metric, and the evaluation result reflects the functionality of each for all metrics.

Similarly, the authors take some coaches as examples to describe the weighted arithmetic mean of comprehensive evaluation. The following table is the final scores of the three coaches (see Table 2).

Here, the authors let the $A 1_{W}, A 2_{W}$, and $A 3_{W}$ denotes the weight of the winning rate, the contribution rate, and the cycle of the honor, respectively.

They take the Mike Krzyzewski for example, the final scores for Mike Krzyzewski can be calculated in the following formula:

$$
F_{M}=A 1 \times A 1_{W}+A 2 \times A 2_{W}+A 3 \times A 3_{W}=0.0208290
$$

Then, we can deal with the rest of the scores of every coach in the same method.

\section{THE BEST ALL TIME COLLEGE COACH IN MODEL 1}

First, the authors standardize the metrics, and get the 
Table 2. Final Scores of the Three Coaches

\begin{tabular}{lllll}
\hline Name & A1 & B1 & C1 & Final scores \\
\hline Mike Krzyzewski & .1052 & .1135 & .0022 & .0208290 \\
Herb Magee & .0984 & .0943 & .0047 & .0203500 \\
Jim Boeheim & .1027 & .1149 & .1178 & .0117686 \\
\hline
\end{tabular}

weight of every metric by using the coefficient of variation method, and finally get the final scores of every coach by using the weighted arithmetic mean of comprehensive evaluation. The following are the coaches in the top eight.

In Figure 1, the blue bar represents the coaching time of the coach, the abscissa denotes the teaching time, and the ordinate denotes the final scores of the coach. The higher the blue bar is, the higher the coach's rank is. They summarize the top five coaches in Model 1 for basketball (see Table 3).

\section{MODEL 2: COACH EVALUATION MODEL FOR ALL GENDERS}

Regardless of the male coaches and the female coaches, they all have the potential and the ability to train a best team, so the authors think they can have the same level of personal ability, what they should consider about is the gender of the team the coach coached, therefore, they should find a way to equal the men's team to the women's team.

They choose to get the balance factor in order to compare them directly. Here, they define four variables, they are $N_{W}, N_{M}, \bar{N}_{W}, \bar{N}_{M}$, the $N_{W}$ and $N_{M}$ represents the total winning number of the women's team and the men's team, similarly, the $\bar{N}_{W}$ and $\bar{N}_{M}$ represents the average winning number of the women's team and the men's team. On account for the difference of the physical quality between men and women, they can give the computation of the balance factor:

$$
\text { blancefactor }=\bar{N}_{M} / \bar{N}_{W}
$$

Since the women's teams are generally in bad situation in comparison with the men's teams. So the balance factor must be larger than one. When dealing with the data, if they meet the data for the women's team, they all let the data multiplied by the balance factor, so that they can regard the women's team as the men's team.

After the discussion of the balance factor, they use Model 1 to solve the problem, and get the following diagram (see Figure 2 and Table 4).

\section{MODEL 3: COACH EVALUATION MODEL FOR ALL GENDERS AND TIMELINE}

With the times past by requirement and development, the understanding of the meaning of the sports is more comprehensive and profound, the ability of the coaches is better. In order to reflect the positive influence of the timeline, here, the authors define another time factor, and the time factor can be expressed by the growth membership function, to reflect the experience brought with the time going by, they use the integral form of the growth membership function, then it can be described as follows:

$$
F_{T}=\left\{\begin{array}{c}
0, x<a \\
\int_{a 1}^{b 1} \frac{x-a}{b-a} d x, a \leq x \leq b \\
1, x>b
\end{array}\right.
$$

Here, the $a$ represents the year of the 1913, the $b$ represents the year of the 2013, and the $a 1$ and $b 1$ represents the start and the teaching time. 


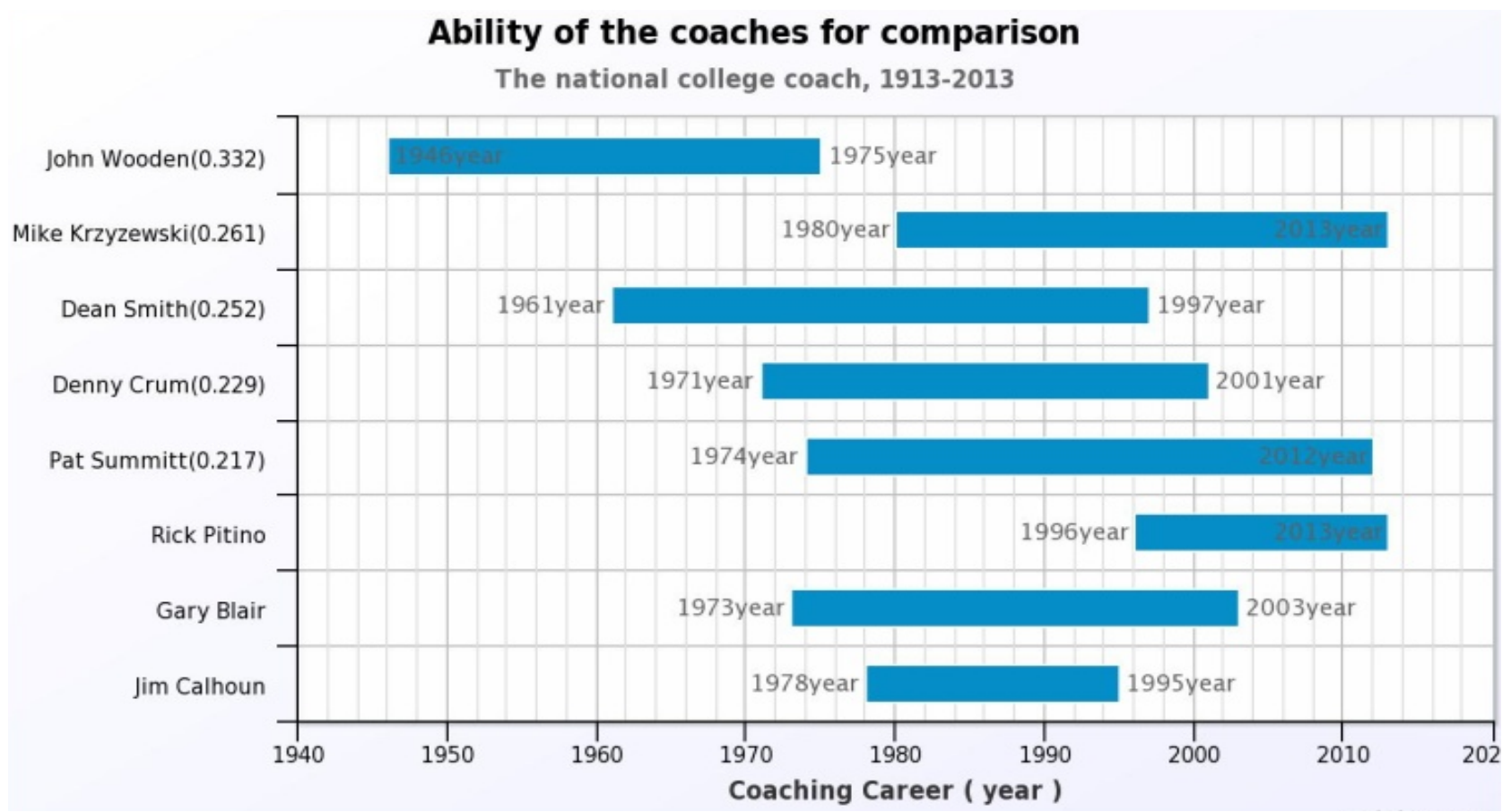

Figure 1. The Best All Time Coach in Model 1.

Table 3. The Top Five Coaches in Model 1

\begin{tabular}{llllll}
\hline Rank & 1 & 2 & 3 & 4 & 5 \\
\hline Gender & Male & Male & Male & Male & Female \\
Name & John Wooden & Mike Krzyzewski & Dean Smith & Denny Crum & Pat Summitt \\
\hline
\end{tabular}

\section{Ability of the coaches for comparison}

The national college coach, 1913-2013

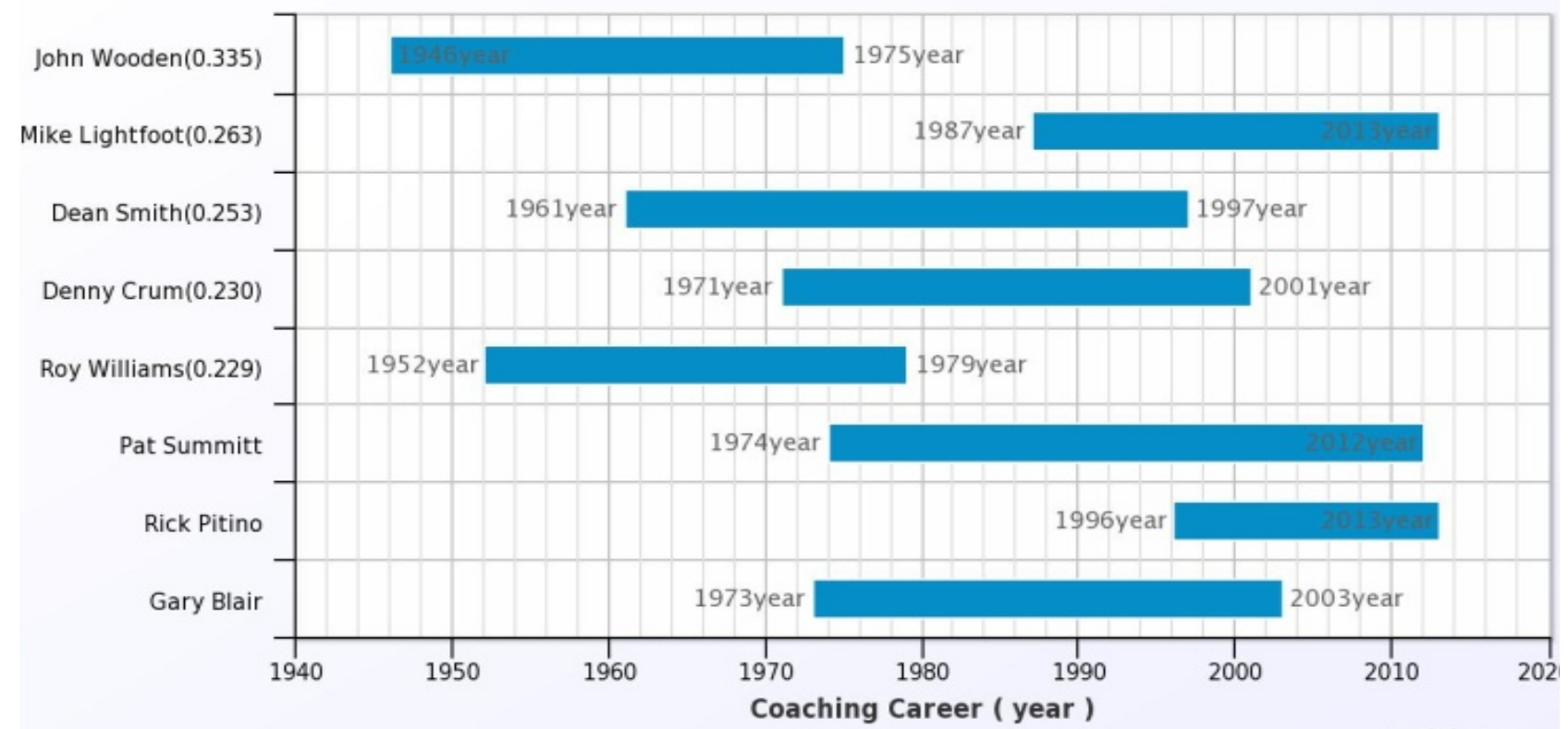

Figure 2. The Best All Time Coach in Model 2. 
Table 4. The Top Five Coaches in Model 2

\begin{tabular}{llllll}
\hline Rank & 1 & 2 & 3 & 4 & 5 \\
\hline Gender & Male & Male & Male & Male & Male \\
Name & John Wooden & Mike Lightfoot & Dean Smith & Denny Crum & Roy Williams \\
\hline
\end{tabular}

Ability of the coaches for comparison

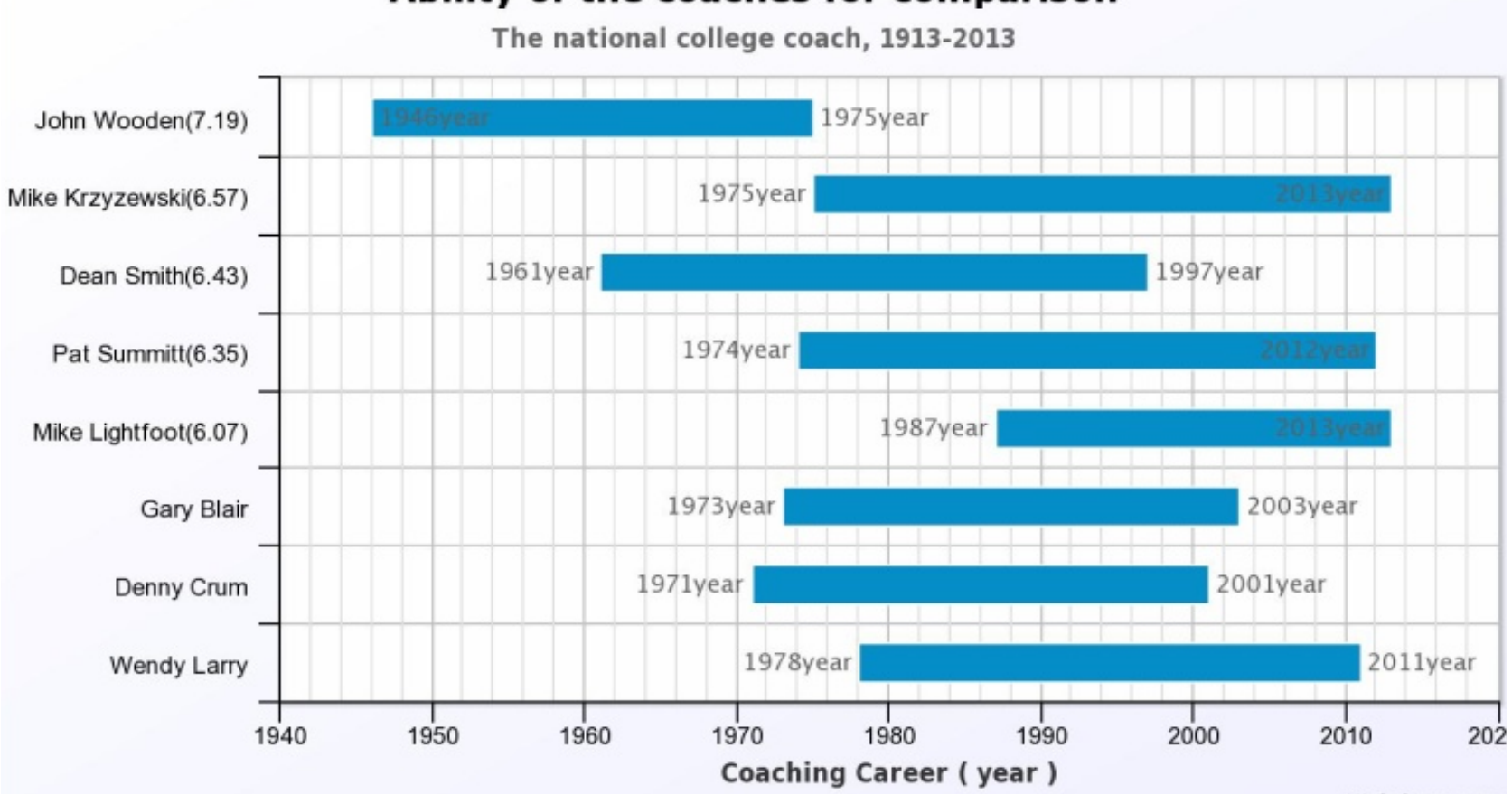

Figure 3. The Best All Time Coach in Model 3.

Table 5. The Top Five Coaches in Model 3

\begin{tabular}{llllll}
\hline Rank & 1 & 2 & 3 & 4 & 5 \\
\hline Gender & Male & Male & Male & Male & Male \\
Name & John Wooden & Mike Krzyzewski & Dean Smith & Pat Summitt & Mike Lightfoot \\
\hline
\end{tabular}

\section{The Final Results in Model 3}

The above are the diagrams of the best all time coach (see Figure 3 and Table 5).

\section{CONCLUSIONS}

It can be seen from Table 5 that the top five coaches evaluated in this paper are John Wooden, Mike Krzyzewski, Dean Smith, Pat Summitt, and Mike Lightfoot. The results are accepted by most people, because the result is related to the reality, and the result is justified in the top 10 basketball coaches. This paper does not discuss about their private lives, whatever their lives are, worthy of praise, or with scandals, we should admit what they do for our sports cause, though the paper gives the five top coaches, we all know that most of the coaches deserve our respect, they love the occupation, and they are cautious and conscientious. With the time going by, the coaches may be written in our books, but they do not leave us alone, they will always inspire us to study, to be more higher, more stronger, and more faster, they will always be in our heart. 


\section{Note}

1. Retrieved (http://www.sojump.com/viewstat/3077481.aspx).

\section{References}

Ni, W. and Y. Bai. 2005. "Construction of Evaluation System of General Ability for the Coaches in Excellent Volleyball Team of China." Journal of Chengdu Sport University 4:69-72.

Zhang, J. 2007. "Basketball Teaching Material and the Construction of Coach's Intellectual System." Journal of Physical Education Institute of Shanxi Normal University 3:49-50
Yu, L. P., Y. T. Pan, and Y. S. Wu. 2015. The Research on the Data Standardization for Comprehensive Evaluation in Academic Journals. Retrieved (http://wenku.baidu.com/link? url=5iSpZlYAkwst4AOrFqNiCB-TDilyrbaCxoooWghGE2 ttwWroUCnkFmoJzEGsuxmd_CYsMLhNHG39JvGdYRX aMihi-e2Svyc3Vv-LMKN2WyO\&qq-pf-to=pcqq.c2c).

\section{Bios}

Xue Yang, College of Electrical Engineering and Automation, North China Electric Power University, China.

Jiwei Jin, College of Electrical Engineering and Automation, North China Electric Power University, China. 